\title{
Performance of Threshold Detection in Cognitive Radio with Improved Otsu's and Recursive One-Sided Hypothesis Testing Technique
}

\author{
P. Venkatapathi, Habibulla Khan, S. Srinivasa Rao
}

\begin{abstract}
Cognitive radio (CR) is a new technology proposed to enhance spectrum efficiency by enabling unlicensed secondary users to access the licensed frequency bands without getting involved with the primary users licensed. Although considered optimal, in order to calculate the signal threshold, this approach requires prior noise statistics information. Even though considered optimal, in order to calculate the signal threshold, this approach requires prior noise statistics information. A prominent example of an Adaptive Threshold Estimation Technique (ATT) for energy detection in Cognitive Radio (CR) is the Recursive One-sided Hypothesis Testing Technique (ROHT). Accurate threshold values are known to be calculated based on the correct choice of their parameter values, which include the standard deviation coefficient and the stop criteria. In this paper, for efficient threshold estimation, the improved Otsu and ROHT are combined for estimating threshold even in the presence of noise floor without need of prior knowledge. The proposed methodology for enactment in cognitive radio sensor networks (CRSN) system based on the adaptive threshold energy detection model with noise variance estimation. The simulation is carried out with the help of Matlab 2017a with the improved Otsu and ROHT techniques. The results obtained shows that improved Otsu and ROHT techniques outperforms that of fixed threshold energy detection in terms of different probability of false alarm rates and miss detections.
\end{abstract}

Index Terms: Adaptive, Cognitive Radio, Energy Detector, Recursive One-sided Hypothesis Testing, Threshold

\section{INTRODUCTION}

Spectrum sensing techniques has received much attention in the last few years, in which radiometer is used to identify the absence or presence of a primary user (PU) in the observed frequency band with the help of simple energy detecting technique. Another purpose of the energy detection method is that it helps to change a threshold for the right decision making. There have been a number of research efforts devoted in the field of measuring noise floor and selecting good thresholds may be used. Almost all examples are addressed in [1-4] of fixed threshold methods.

While the fixed threshold methods are easily scalable, because of presence of noise variation that makes erroneous decision-making. This results in a higher false alarm and miss detection rates. Towards this reason, CRSN applications are more beneficial for adaptive and autonomous threshold

Revised Manuscript Received on July 22, 2019

P. Venkatapathi, Research Scholar, Department of ECE, KLEF, Vijayawada, Andhra Pradesh, India.

Dr. Habibulla Khan, Professor, Department of ECE, KLEF, Andhra Pradesh, India

Dr. S. Srinivasa Rao, Professor \& HOD, Department of ECE, MRCET, Hyderabad, Telangana, India. methods. Several countermeasures have been investigated and used to alleviate misdetection rate. The studies of adaptability can be obtained by evaluating the statistic characteristics of the obtained spectrum measures, as the standard deviation provides a medium significance for the spectral information dispersion. In [5-9] some of the adaptive and automatic threshold techniques were addressed. While the above methods require prior knowledge, in this work we describe three algorithms that assess the threshold from the direct data without needing prior knowledge. The main contributions and organization of this paper are summarized as follows: In section 2 we describe background details of Energy detection. Section 3 system model. Section 4 discusses the proposed work. Section 5 deliberates results and discussions. Finally, in section 6 , we concluded the paper.

\section{BACKGROUND WORK}

The ideal method for the identification of signals samples depending on energy measurement is energy detection technique [11] among the different techniques of signal detection mentioned in literature [10]. The primary purpose is to test whether the energy of signal approaches the set threshold value otherwise it is treated as noise floor. In order to adapt their respective threshold values according to different channel conditions, new ED designs are required. This led to the design with several adaptive threshold estimation methods (ATT) in the literature [12-15], based on Recursive One-Sided Hypothesis Testing (ROHT) technique being the most viable algorithms for use in the ED [16], [17].

The ROHT [16-19] is known for its simplicity, efficiency and effectiveness. Furthermore, it is and has always been an undiscovered exercise in the literature to determine the performance limits of the ROHT algorithm with respect to its minimum signal to noise ratio (SNR) level. This knowledge will allow users to determine specific conditions that may no longer guarantee the performance of the ROHT.

\section{SYSTEM MODEL}

The absence of knowledge of noise levels can restrict or even limits the processing ability of the energy detector (ED) in the cognitive node. For the reason that the energy detector has no previous knowledge of the primary user (PU) or noise floor in the system. We put forward using the recursive ROHT algorithm to estimate channel noise variance and SNR. The main advantage of this technique is its convenience in calculating 
the SNR value and corresponding decision of threshold value. This characteristic creates the potential relative to the other previously mentioned adaptive threshold techniques for noise estimation in cognitive sensor nodes. One of the simplest form of Otsu's algorithm is its optimum threshold responsible for getting histogram properties with less involvement of pixel values as discussed in [11]. For obtaining two classes, measure the threshold optimum value for signal and noise. Apply the argument data is measured into $L$ levels with values $s \times[1,2, \ldots L]$. Consider $g_{i}$ be treated as $i^{\text {th }}$ gray level value, $p_{i}$ treated to be probability level.

The distribution mean is defined as:

$\mu_{T}=\sum_{i=1}^{L} g_{i} p_{i}$

Appling argument, $T=g_{k}$, employed for separating the probability distribution into two major classes the primary is noise class $C_{1}$ and the secondary is signal class $C_{2}$, by corresponding levels $[1,2, . . k] \in$ and $C_{1}$ levels $[k+1, . . L] \in$ $C_{2}$. The above argument also can define as number the of points with the gray level at $i$ is denoted by $x_{i}$ and the entire number of points can be expressed as

$X=x_{1}+x_{2}+\ldots+x_{L}$

So the histogram of the data is considered as an occurrence distribution of probability

$$
p_{i}=\frac{x_{i}}{X}, x_{i} \geq 0, \sum_{i=1}^{L} x_{i}=1
$$

For obtaining distribution of gray level with the help of classes are symbolically shown as

$$
\begin{aligned}
C_{1} & =\frac{p_{1}}{\sum_{i=1}^{k} p_{i}}, \frac{p_{2}}{\sum_{i=1}^{k} p_{i}}, \ldots \ldots, \frac{p_{k}}{\sum_{i=1}^{k} p_{i}} \\
C_{1} & =\frac{p_{k+1}}{\sum_{i=k+1}^{L} p_{i}}, \frac{p_{k+2}}{\sum_{i=k+1}^{L} p_{i}}, \ldots . ., \frac{p_{L}}{\sum_{i=k+1}^{L} p_{i}}
\end{aligned}
$$

Also, the means for classes $C_{1}$ and $C_{2}$ are:

$$
\begin{array}{r}
\mu_{1}=\frac{\sum_{i=1}^{k} i_{1} p_{i}}{\sum_{i=1}^{k} p_{i}} \\
\mu_{2}=\frac{\sum_{i=k+1}^{L} i \cdot p_{i}}{\sum_{i=k+1}^{L} p_{i}}
\end{array}
$$

Consider $\mu_{T}$ total data mean value so that it can be summed together to gives.

$$
\beta_{1} \mu_{1}+\beta_{2} \mu_{2}=\mu_{T}
$$

Where

$$
\beta_{1}=\sum_{i=1}^{k} p_{i}
$$

And

$$
\beta_{2}=\sum_{i=k+1}^{L} p_{i}
$$

As per observations, the probabilities summation is equal to one.

$$
\beta_{1}+\beta_{2}=1
$$

Finally, Otsu defined the between-class variance two classes $C_{1}$ and $C_{2}$ as:

$$
\sigma^{2}=\beta_{1}\left(\mu_{1}-\mu_{T}\right)^{2}+\beta_{2}\left(\mu_{1}-\mu_{T}\right)^{2}
$$

By technique so called bi-level thresholding, that the optimal threshold $t$ is measurable quantity capable of maximizes between-class variance

$$
t^{\prime}=\max \left\{\sigma^{2}(t)\right\}, 1 \leq t \leq L
$$

\section{PROPOSED WORK}

The threshold is estimated with the application of a unilateral hypothesis test or the Otsu algorithm. By definition threshold is used to find a proportion of the data as signal samples and the rest as noise floor. For remaining unclassified measurements, the signal portion is rejected and repeated iteratively until the change between two consecutive iterations in the standard deviation (SD) of unclassified data is equal or below $\epsilon$, where $\epsilon$ is an arbitrary positive value which can be determined.

\section{(Algorithm1) Improved Otsu's algorithm:}

If the $C_{k}(k=0$ or 1$)$ distribution is skewed, it is known that the average value is a very resilient estimate connected to the average gray level. Therefore we find that the average replacement of the median value can obtain $t^{\prime}$ which results in a heavily tailed distribution for $C_{k}$ more exact than those selected by the usual Otsu algorithm. In the entire gray level, we can replace the total mean $\mu_{T}$ with the total median level $m_{T}$ of all points. Similar to the whole data $\mu_{T}$ mean value, the mean $\mu_{1}$ and $\mu_{2}$ value of the signal parts $C_{l}$ and $C_{2}$ noise parts can be replaced with the median gray-level $m_{1}$ and $m_{2}$, respectively.

The between-class variance $\sigma^{2}$ of the part $C_{1}$ and $C_{2}$ can be rewritten as

$$
\sigma^{2}=\beta_{1}\left(m_{1}-m_{T}\right)^{2}+\beta_{2}\left(m_{2}-m_{T}\right)^{2}
$$

And the goodness threshold $t^{\prime}$ is chosen by maximizing

$t^{\prime}=\max \left\{\sigma^{2}(t)\right\}, 1 \leq t \leq L$

(Algorithm2) Improved ROHT algorithm: The recurrent Otsu algorithms vary from the ROHT because it now measures the thresholds and to use the Otsu algorithm instead of the one-sided ROHT algorithm. The contribution to the model is the $p_{o}$ value the repetitive Otsu method suggested. In such techniques, the previous notes are often used:

- Denote $M$ to be pool of samples for measurement,

- Denote $S$ be the pool of signals inside $M$ samples,

- $S_{\mathrm{k}}$ be a subset of $S$ for the $k^{\text {th }}$ iteration of the algorithm,

- Consider $Q$ be the pool of noise samples within $M$,

- Consider $Q_{k}$ be a superset of $Q$ for the $k^{\text {th }}$ iteration of the algorithm, $Q_{k}$ may comprise signals,

- $\mu_{k}, \sigma_{k}=$ mean and standard deviation of the elements of $Q_{k}$, and

- $\theta_{k}=$ Decision threshold for the $k^{\text {th }}$ iteration to define the original signal part. 


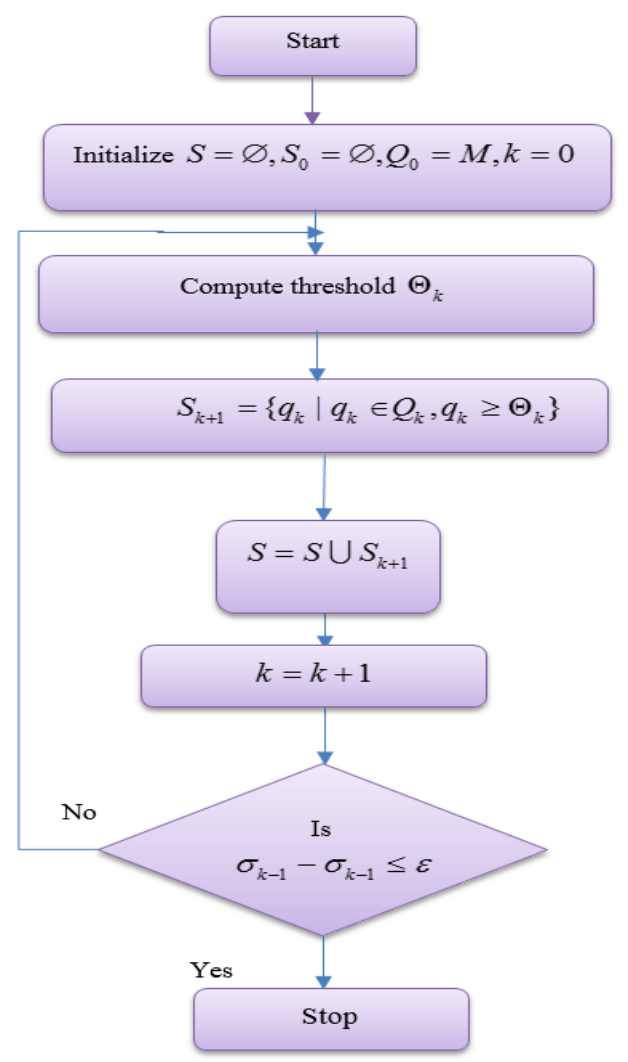

Figure. 1: Flowchart for recursive thresholding

\section{RESULTS \& DISCUSSION}

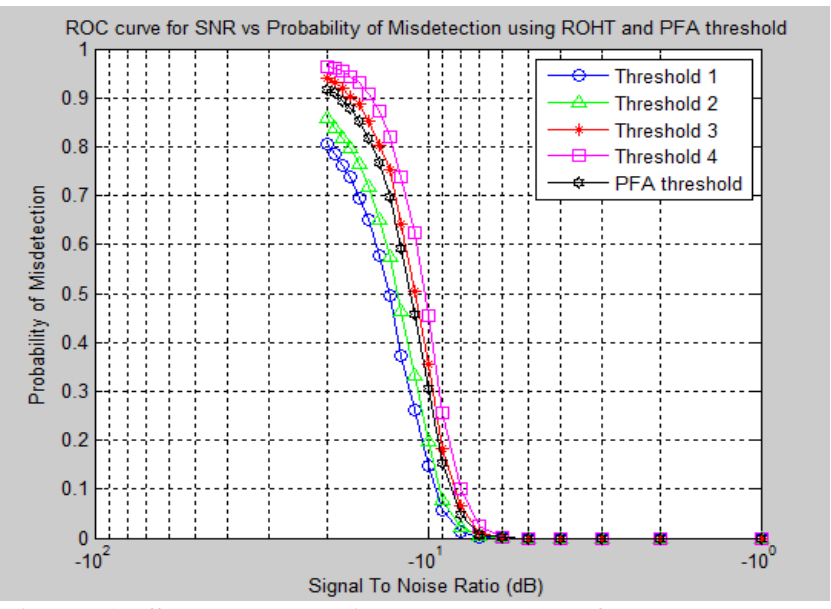

Figure.2: SNR vs $\boldsymbol{P}_{m d}$ using Improved ROHT thresholds in comparison to the fixed threshold conventional energy detector for $\boldsymbol{P}_{f}=\mathbf{0 . 0 1}$

We simulate and analyze with further precision the traditional energy detection technique. We consider the SNR range from $-20 \mathrm{~dB}$ to $0 \mathrm{~dB}$ and the chance of false alarm of 0.1 and 0.01 in this simulation. In addition, 1000 samples of the signal received and 10,000 simulations from Monte Carlo are regarded. The signal received is based on the primary signal and White Additive Gaussian Noise (AWGN). Figure.2 shows that the likelihood of misdetection varies depending on the SNR. This shows from the fact that an energy detector (ED) have detection probability to be higher false alarm to be lower when SNR approaches $-7 \mathrm{~dB}$. This means that the probability of error detection increases as the SNR decreases.
This is the general trend in the adaptive technique based ED and the conventional ED.

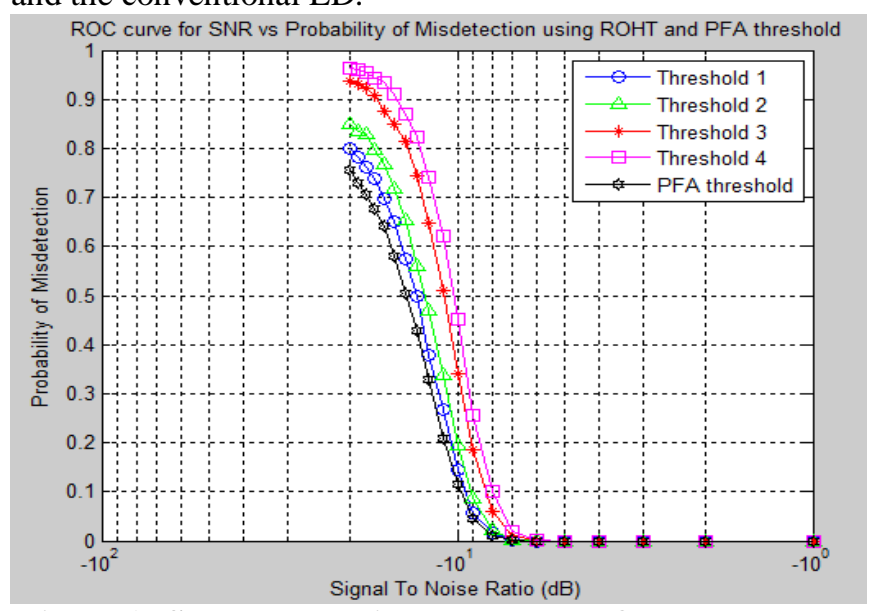

Figure.3: SNR vs $\boldsymbol{P}_{m d}$ using Improved ROHT thresholds in comparison to the fixed threshold conventional energy detector for $\boldsymbol{P}_{f}=\mathbf{0 . 1}$

\section{CONCLUSION}

The ROHT technology comes from the statistical world, where it is first conveyed as the one-sided test for the hypothesis. Depending on its statistical properties, our proposed algorithm relates the original signal with that of noise floor of received signal. In this paper, we address the influence of direct threshold estimation without need of threshold prior knowledge generated considerable attention in terms of manual involvement to be less extent. It was shown that improved ROHT have higher SNR values as compared to the fixed threshold energy detector.

\section{ACKNOWLEDGMENT}

Authors would like to express sincere gratitude to management and principal of KLEF, Vijayawada, Andhra Pradesh, India, for their support and encouragement to carry out the research work.

\section{REFERENCES}

1. M. H. Islam, C. L. Koh, S. W. Oh, X. Qing, Y. Y. Lai, C. Wang, Y.-C Liang, B. E. Toh, F. Chin, G. L. Tan, and W. Toh, "Spectrum Survey in Singapore: Occupancy Measurements and Analyses," in 3rd International Conference on Cognitive Radio Oriented Wireless Networks and Communications (Crowcon 2008), 2008, pp. 1-7.

2. R. I. C. Chiang, G. B. Rowe, and K. W. Sowerby, "A Quantitative Analysis of Spectral Occupancy Measurements for Cognitive Radio," in IEEE 65th Vehicular Technology Conference - VTC2007-Spring, 2007, pp. 3016-3020.

3. R. Gonzalez and R. Woods, Digital image processing, 3rd ed. Pearson Prentice Hall, 2002.

4. W. K. Pratt, Digital Image Processing, 3rd ed., vol. 5. John Wiley \& Sons, Inc., 2001.

5. S. D. Barnes, P. A. Jansen Van Vuuren, and B. T. Maharaj, "Spectrum occupancy investigation: Measurements in South Africa," Measurement: Journal of the International Measurement Confederation, 2013.

6. S. V Vaseghi, Advanced Digital Signal Processing and Noise Reduction, 4th ed. John Wiley \& Sons, Inc., 2008.

7. D. Datla, A. M.Wyglinski, and G. J. Minden, "A Spectrum Surveying Framework for Dynamic Spectrum Access Networks," IEEE Transactions on Vehicular Technology, vol. 58, no. 8, pp. 4158-4168, 2009.

8. F. Weidling, D. Datla, V. Petty, P. Krishnan, and G. J. Minden, “A framework for R.F. spectrum measurements and analysis," in First IEEE International Symposium on New Frontiers 


\section{Testing Technique}

in Dynamic Spectrum Access Networks, 2005. DySPAN 2005. 2005, pp. 573-576.

9. D. Datla, A. Wyglinski, and G. Minden, "A Statistical Approach to Spectrum Measurement Processing," Information and Telecommunication Technology Center, 2005.

10. D. Cabric, S. M. Mishra, and R. W. Brodersen, "Implementation Issues in Spectrum Sensing for Cognitive Radios," in Conference Record of the Thirty-Eighth Asilomar Conference on Signals, Systems and Computers, vol. 1, (Pacific Grove, CA, USA), pp. 772- 776, November 2004

11. R. Tandra and A. Sahai, "Fundamental Limits on Detection in Low SNR Under Noise Uncertainty," in International Conference on Wireless Networks, Communications and Mobile Computing, vol. 1, pp. 464-469, 2005.

12. C. Liu, M. Li, and M.-L. Jin, "Blind energy-based detection for spatial spectrum sensing," IEEE Wireless Communications Letters, vol. 4, no. 1, pp. 98-101, feb 2015.

13. B. K. Jariwala and V. R. Manilal, "A Survey: A Cognitive Radio for Wireless Communication," IOSR Journal of Electronics and Communication Engineering, vol. 9, no. 1, pp. 57-63, 2014.

14. R. K. Dubey and G. Verma, "Improved spectrum sensing for cognitive radio based on adaptive double threshold," International Journal of Emerging Trends in Electrical and Electronics (IJETEE), vol. 11, no. 2, pp. 1-6, 2015.

15. A.Chavez, R. Littman-Quinn, K. Ndlovu, and C. L. Kovarik, "Using TV white space spectrum to practice telemedicine: A promising technology to enhance broadband internet connectivity within healthcare facilities in rural regions of developing countries," Journal of telemedicine and telecare, vol. 22, no. 4, pp. 260-263, 2016.

16. D. Datla, A. M. Wyglinski, and G. J. Minden, "A statistical approach to spectrum measurement processing," Information and Telecommunications Technology, 2007.

17. D. Datla, A. M. Wyglinski, and G. Minden, "A spectrum surveying framework for dynamic spectrum access networks," IEEE Transactions on Vehicular Technology, vol. 58, no. 8, pp. 4158-4168, 2009.

18. A.J. Onumanyi, E. N. Onwuka, A. M. Aibinu, O. C. Ugweje, and M. J. Salami, "A modified Otsu's algorithm for improving the performance of the energy detector in cognitive radio," AEU - International Journal of Electronics and Communications, vol. 79, pp. 53-63, 2017.

19. J. Onumanyi, E. N. Onwuka, A. M. Aibinu, O. C. Ugweje, and M. J. E. Salami, "A real valued neural network based autoregressive energy detector for cognitive radio application," International Scholarly Research Notices, vol. 2014, pp. 1-11, 2014.

\section{AUTHORS PROFILE}

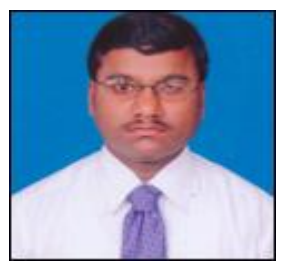

Venkatapathi Pallam, received M.Tech Degree from JNTU Hyderabad, Telangana, India in 2009. $\mathrm{He}$ is currently working toward the Ph.D. degree in electronics and communication engineering, $\mathrm{K}$ L University, Vijayawada, AP, India. Also working as an Assistant Professor at Malla Reddy College of Engineering, Secunderabad, Telangana, India. He has 14 years of experience in the field of teaching. His area of research Interest includes Wireless communication \& signal processing.

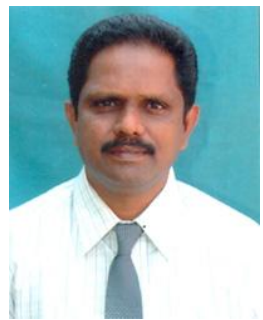

Dr Habibulla Khan, received Ph.D degree from Andhra University in the year of 2007. Presently working as Professor and dean students affaire in the KL University. He published various national and international journals \& conferences. He is a member of professional bodies like IEEE, ISTE and IETE. His area of research Interest includes wireless communications \& signal processing. He published more number of papers in various national and international journals \& conferences. His area of research Interest includes wireless communications, signal processing and antennas.

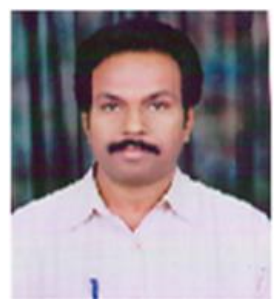

Dr. S. Srinivasa Rao, received the B.Tech degree from Madras Institute of Technology, Anna University, and the M.Tech and $\mathrm{Ph}$. D from JNTU Hyderabad, Telangana, India. Presently working as Professor and Head of the Department at Malla Reddy College of Engineering and Technology, Secunderabad. He has 26 years of experience in the field of teaching. He is a member of professional bodies like IEEE, ISTE and IETE and also reviewer for springer's international journal on wireless personal communication. $\mathrm{He}$ published more number of papers in various national and international journals \& conferences. His area of research Interest includes wireless communications \& signal processing. 